\title{
Simulation of the Motion of Oil Ganglia in Consolidated Porous Media. Crowding Effects
}

\author{
VALAVANIDES, MARIOS S. \\ CONSTANTINIDES, GEORGE N. \\ PAYATAKES, ALKIVIADES C. \\ Department of Chemical Engineering, University of Patras, and \\ Institute of Chemical Engineering \& High Temperature Chemical Processes, Greece
}

Paper presented at the 5th European Conference on the Mathematics of Oil Recovery, Leoben, Austria, 3-6 Sept. 1996

\section{ABSTRACT}

Oil ganglion dynamics (that is, the collective flow behavior of a population of interacting oil ganglia in a porous medium) arises in connection with oil bank formation and maintenance during enhanced oil recovery with immiscible flooding. Furthermore, recent experimental observations have shown that ganglion dynamics is involved in steady-state two-phase flow, and therefore it is central to the quantitative analysis of the relative permeabilities. A recently developed computeraided three-dimensional network simulator can be used to predict the cooperative flow behavior of dense populations of oil ganglia moving through consolidated porous media. The differences between the flow behavior of a solitary ganglion and that of a ganglion that is a member of a dense population (at various values of oil saturation and viscosity ratio) are examined. The ganglion velocity, the stranding and breakup coefficients and the mode of breakup are calculated as functions of the ganglion volume, the oil saturation and the viscosity ratio, keeping all other parameters fixed at typical values.

\section{INTRODUCTION}

Oil ganglion population dynamics play a crucial role in a number of important two-phase flow processes, including waterflooding of oil reservoirs, immiscible stages of enhanced oil recovery (EOR), steady-state twophase flow in porous media, etc. Here, we use a recently developed simulator (Constantinides \& Payatakes, 1996) to study the effects of the oil saturation and other system parameters on the flow behavior of ganglia.
Originally, ganglion dynamics was conceived as a means of analyzing EOR immiscible flooding processes (Payatakes et al., 1980). Most of the work that followed was in the same frame (Payatakes, 1982; Payatakes and Dias, 1984; Constantinides and Payatakes, 1991; Sahimi, 1993). However, recent experimental (Avraam and Payatakes, 1995; Avraam et al., 1994) and theoretical works (Constantinides \& Payatakes, 1996; Valavanides et al., 1996) have shown that ganglion dynamics is one of the main flow regimes of steady state two phase flow in porous media.

A parametric experimental study of the pore level mechanisms of steady-state two-phase flow through chamber-and-throat pore networks (etched-glass models) has shown that over broad ranges of the values of the main dimensionless parameters (capillary number, $\mathrm{Ca}$, oil/water viscosity ratio, $\kappa$, the imposed oil/water flowrate ratio, $r$, saturation of the aqueous phase, $S_{w}$ ) the oleic phase is disconnected in ganglia or drops and the oil flowrate is mainly or totally due to the motion of ganglia and/or oil drops (Avraam and Payatakes, 1995; Avraam et al., 1994). The oil motion can be described as a continual process of ganglion mobilization, collision and coalescence, breakup, stranding, remobilization, etc., resulting in an overall dynamic equilibrium denoted as steady-state ganglion dynamics. The flow regime and the corresponding relative permeabilities are strong functions of $\mathrm{S}_{\mathrm{w}}, \mathrm{Ca}, \kappa, \mathrm{r}$, the coalescence factor, $\mathrm{Co}$, the wettability (cosines of the dynamic contact angles $\theta_{\mathrm{a}}$ and $\theta_{\mathrm{r}}$ ), the geometry and topology of the pore network), and the history of the experiment (wetting saturation increasing or decreasing). Moreover, for a given porous medium, the steady-state relative permeabilities correlate strongly with the corresponding flow regime. Therefore, understanding 
ganglion population dynamics is also of fundamental value in analyzing steady-state two-phase flow processes.

In the present work, we will focus our attention on the cooperative behavior of interacting ganglia, when they become mobilized. Melrose and Brandner (1974) developed a criterion for solitary ganglion stranding/remobilization, which was extended and generalized by $\mathrm{Ng}$ and Payatakes (1980) (see also Payatakes and Dias, 1984). The motion and fate of solitary oil ganglia has been studied by several researchers both experimentally $(\mathrm{Ng}$ et al., 1978; Rapin, 1980; Legait, 1983; Legait et al., 1983; Hinkley et al., 1987) and theoretically ( $\mathrm{Ng}$ and Payatakes, 1980; de la Cruz and Spanos, 1983; Legait, 1983; Legait et al., 1983; Dias and Payatakes, 1986b; Constantinides and Payatakes, 1991). All these studies have shown that mobilization of solitary ganglia takes place for relatively high $\mathrm{Ca}$ values $\left(\mathrm{Ca} \geq 10^{-4}\right)$. However, the aforementioned experimental results of steady-state two-phase flow (Avraam and Payatakes, 1995) have shown that, even for relatively low Ca values, say $\mathrm{Ca} \sim 10^{-8}-10^{-7}$, a significant portion of the ganglion population is mobilized for a broad range of oil saturation values $\left(0.3<S_{o}<0.7\right)$. It should be noted here that the pertinent dimensionless parameter for ganglion mobilization is the ganglion mobilization number, $\mathrm{Gm}$ ( $\mathrm{Ng}$ and Payatakes, 1980; Payatakes and Dias, 1984). Gm is the ratio of the viscous stresses which tend to mobilize the ganglion to the capillary pressure opposed to the motion. When $\mathrm{Gm} \geq \mathrm{l}$ the ganglion becomes mobilized. The calculation of $\mathrm{Gm}$ should be based on the value of $\mathrm{Ca} / \mathrm{k}_{\mathrm{rw}}$, where $\mathrm{k}_{\mathrm{rw}}$ is the local and instantaneous relative permeability to water $(\mathrm{Ng}$ and Payatakes 1980). When $k_{\mathrm{rw}}$ is sufficiently small, Gm may be larger than unity for many ganglia, even if the capillary number is small. Therefore, these ganglia become mobilized.

A computer-aided three-dimensional microflow simulator of steady-state ganglion population dynamics in consolidated porous media was developed by Constantinides and Payatakes (1996). Pore-scale models (Ng and Payatakes 1980; Payatakes et al., 1980; Dias and Payatakes 1986a, 1986b; Constantinides and Payatakes 1991) are used to simulate the complex flow phenomena at pore level and the dynamic flow behavior of each individual ganglion. The simulator calculates ganglion population interactions and predicts the flow regime on the mesoscopic scale and the relative permeabilities in agreement with reported experimental results (Avraam and Payatakes, 1995). This simulator is used here to predict the flow behavior of individual ganglia which are members of a dense ganglion population. The simulator is also used to study the flow behavior of solitary ganglia. The flow behavior of the ganglia is mainly characterized by the ganglion velocities, the breakup and stranding coefficients and the mode of ganglion breakup, which are calculated as functions of the oil saturation and the pertinent dimensionless parameters.

A novel analytical theory for the macroscopic description of two-phase flow in porous media, when the dominant flow regime is ganglion dynamics, was proposed recently (Valavanides et al., 1996). This theory utilizes the ganglion population balance equations (Payatakes, 1982) to predict the macroscopic behavior of the process. To achieve this, the theory utilizes information on the cooperative flow behavior of ganglia in the form of system factors. The results of the present work can be used by this theory directly.

\section{MODEL FORMULATION}

A full description of the microflow network simulator is given in the original reference (Constantinides and Payatakes, 1996). The main features of this simulator are described below for the sake of completeness.

The porous medium model used by the simulator is a three-dimensional network of unit cells of the constricted-tube type, suitable for consolidated porous media (Constantinides and Payatakes, 1989). The chamber and throat diameter distributions of the pore network are typical Berea sandstone pore size distributions (Dullien and Dhawan, 1975). There is a positive correlation of the diameters of the contiguous chambers and throats (Constantinides and Payatakes, $1989 ; 1996)$. We used a pore network of $30 \times 20 \times 5$ nodes ( 3000 chambers and $\sim 9000$ throats). The porosity of the porous medium model is $\varepsilon=0.22$, the mean node-to-node distance is $k 73.1 \mu \mathrm{m}$, and the characteristic void space, that is, the mean volume of a chamber and its adjacent half throats (Conceptual Elemental Void Space) is $\mathrm{V}_{\mathrm{CEVS}}=8610 \mu \mathrm{m}^{3}$.

Initially the pore network is filled with water. Ganglia of preselected volumes and random shapes are placed in random sites in the network, until a preselected value of oil saturation, $S_{o}$, is reached. Then a macroscopic pressure gradient is applied along the network, so that the steady-state capillary number, $\mathrm{Ca}$, takes a preselected value. The value of the macroscopic pressure gradient is determined iteratively. Then, the ganglia are let to move. Under creeping flow conditions, the two-phase flow problem is solved with standard electrical network 
analysis (Dias and Payatakes, 1986a; Constantinides and Payatakes, 1991; 1996; Vizika et al., 1994). The boundary conditions on the sides of the pore network parallel to the direction of the macroscopic flow are assumed periodical. Ganglia leaving the network downstream reenter into the network upstream, to impose a kind of periodicity in the direction of the macroscopic flow, and to keep the oil saturation, $S_{o}$, constant (Constantinides and Payatakes, 1996).

When the displacement starts, some of the ganglia become trapped where they were placed initially, whereas the rest begin to migrate downstream. Due to the randomness of the porous medium, the flow conditions may be significantly different from place to place, even if the capillary number is constant. For this reason, moving ganglia undergo either quasi-static or dynamic displacement (Payatakes and Dias, 1984). Moving ganglia break into daughter ganglia, the smaller of which become stranded where they are formed, or after a few steps. Ganglion breakup occurs either due to pinch-off or dynamic breakup (Payatakes and Dias, 1984). A stranded ganglion may become remobilized, if the local pressure field is changed by the flow of a moving ganglion in the nearby area. Moving ganglia collide with other moving ganglia and coalescence with them with probability, Co, (here, $\mathrm{Co}=0.15$ ) producing new large ganglia. The number of collisions per time step depends of the value of $\mathrm{S}_{\mathrm{o}}$. The combination of breakup, stranding, remobilization and coalescence results in an overall dynamic equilibrium, namely, steady-state ganglion dynamics. Under steady-state conditions, each ganglion is identified and its migration is recorded, until it breaks, becomes stranded, or coalesces with another ganglion. Each simulation lasts for a sufficiently long time interval to obtain statistically reliable results.

Fig. 1 shows two successive snapshots of the cooperative flow behavior of a dense ganglion population $\left(\mathrm{Ca}=10^{-4}\right.$, $\kappa=1.45, C_{0}=0.15, S_{w}=0.5, \theta_{a}^{0}=45^{\circ}$, and $\theta_{r}^{0}=35^{\circ}$ ). The dimensionless time interval between two snapshots is $\Delta t^{*}=\Delta t U_{w} / / \varepsilon=1$, where $U_{w}$ is the superficial velocity of water. For simplicity, a two-layer pore network of size $30 \times 20 \times 2$ is used for this specific simulation. Each chamber is depicted as a sphere of the appropriate diameter, whereas each throat as a cylinder. For better clarity, the node-to-node distance is shown dilated by $50 \%$. Oil is shown white, and water is shown gray.

The simulation of the fate of a solitary ganglion is done in a similar way (see also Dias and Payatakes, 1986b). A ganglion of preselected volume and random shape is placed at a random site of the pore network away from the edges. Then, an appropriate macroscopic pressure gradient is applied, so that the capillary number takes a preselected value. The ganglion migration is followed and recorded, until the ganglion either breaks or becomes stranded.

\section{RESULTS AND DISCUSSION}

The differences of the flow behavior of solitary ganglia and the ganglia that are members of an interacting ganglion population are examined. The flow behavior of ganglia is mainly characterized by the ganglion velocity, $u$, the coefficients of stranding and breakup, $\lambda$ and $\phi$ respectively, and the mode of ganglion breakup, W $\Delta \mathrm{v}$ (Payatakes, 1982). All these variables are functions of the system parameters and the ganglion volume, shape and orientation, the specific flowpath that the ganglion follows (local geometry) and the distribution of the two fluids in the nearby area. For fixed values of the system parameters, $u, \lambda, \phi$ and $\mathrm{W} \Delta \mathrm{v}$ are calculated as functions of the reduced ganglion volume $\left(v^{*}=v / V_{C E V S}\right)$ by averaging the results for different simulations in which the initial ganglion shape, orientation and location in the pore network are changed (Dias and Payatakes, 1986b). In this work $\mathrm{u}, \lambda, \phi$ and $W \Delta v$ are studied as functions of the saturation and the viscosity ratio, as well. All simulations were performed for a constant $\mathrm{Ca}$ value $\left(\mathrm{Ca}=10^{-4}\right)$ appropriately selected to induce motion of solitary ganglia, and to be in the range of practical interest. The wettability (incipient advancing and receding contact angles, $\theta_{\mathrm{a}}^{0}$ and $\theta_{\mathrm{r}}^{0}$ ) and the coalescence factor, $C o$, were kept constant $\left(\theta_{\mathrm{a}}^{0}=45^{\circ}, \theta_{\mathrm{r}}^{0}=35^{\circ}\right.$, and $\mathrm{Co}=0.15$ ) and the porous medium fixed (typical Berea sandstone).

\section{Ganglion velocities}

Ganglion velocity plays a crucial role in ganglion population dynamics (Payatakes, 1982; Payatakes and Dias, 1984; Valavanides et al., 1996). The velocity of a ganglion, $\mathrm{u}$, changes continuously as the ganglion migrates downstream, due to the converging-diverging character of the flow channels and the high degree of randomness of the porous medium (Dias and Payatakes, 1986b; Hinkley et al., 1987). Hence, ganglion velocity is expressed in terms of the time-averaged velocity with which the centroid of a ganglion moves, $\overline{\mathbf{u}}$. Then, for ganglia of a given volume $\mathrm{v}^{*}$, the mean time-averaged ganglion velocity, $\langle\overline{\mathbf{u}}\rangle$, is calculated as the average of the velocities of all $\mathrm{v}$-ganglion migrations considered (see above). In reduced form, the mean time-averaged ganglion velocity is expressed as $\mathrm{u}^{*}=<\overline{\mathrm{u}}>\varepsilon / \mathrm{U}_{\mathrm{w}}$ and is the 

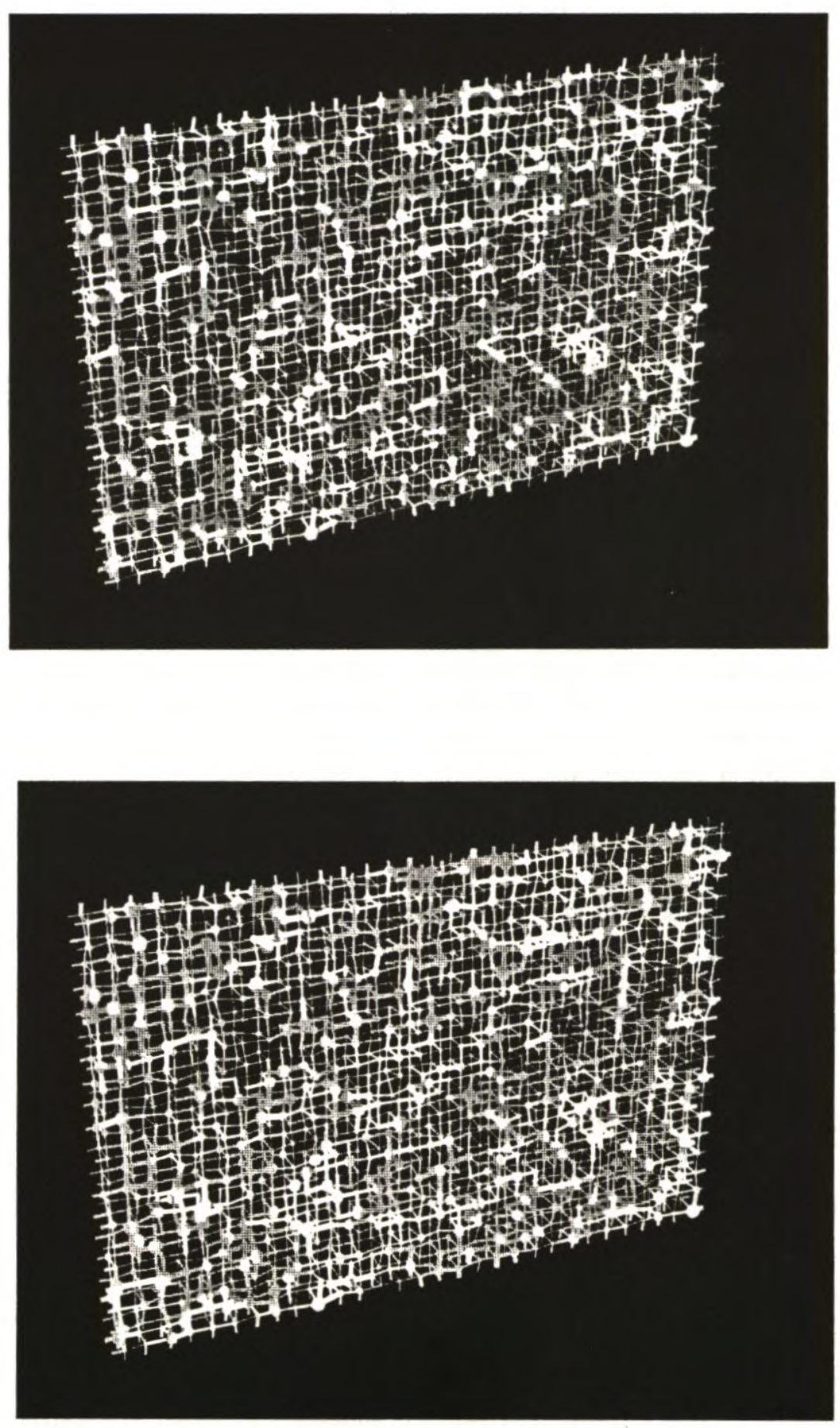

Figure 1: Two successive snapshots of the simulation of the motion of a population of interacting ganglia, for $\mathrm{S}_{\mathrm{w}}=0.5$, $\mathrm{Ca}=10^{-4}, \kappa=1.45, \mathrm{Co}=0.15, \theta_{\mathrm{a}}^{0}=45^{\circ}$, and $\theta_{\mathrm{r}}^{0}=35^{\circ}$ (see text). 

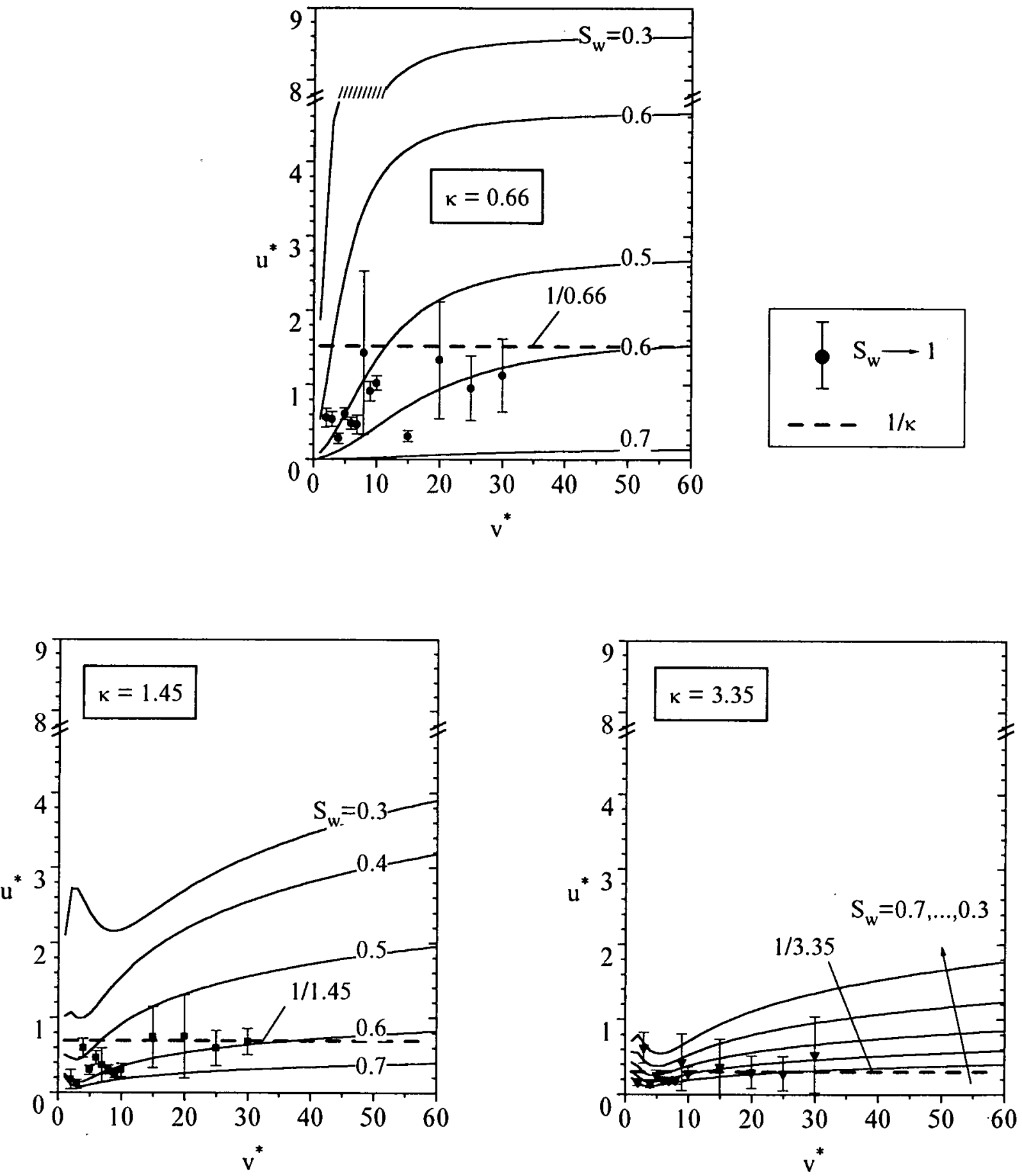

Figure 2: Typical results of the reduced mean time-averaged ganglion velocity, $u^{*}\left(v^{*}\right)$, for solitary ganglia $\left(S_{w} \rightarrow 1\right.$; the data points and the corresponding confidence intervals at confidence level $95 \%$ are shown) and ganglia that are members of an interacting ganglion population $\left(0.3 \leq \mathrm{S}_{\mathrm{w}} \leq 0.7\right.$; the fitting curves are shown), for three typical $\kappa$ values, that is, for $0.66,1.45$ and $3.35\left(\mathrm{Ca}=10^{-4}, \mathrm{Co}_{0}=0.15, \theta_{\mathrm{a}}^{0}=45^{\circ}\right.$, and $\left.\theta_{r}^{0}=35^{\circ}\right)$. 
relative velocity of the ganglia to the interstitial velocity of water $\mathrm{U}_{\mathrm{w}} / \varepsilon$ for an oil free pore network.

For solitary ganglia $\left(\mathrm{S}_{\mathrm{w}} \rightarrow \mathrm{I}\right)$, the calculated reduced mean time-averaged ganglion velocities are shown in Fig. 2 as data points with the respective confidence intervals (confidence level $95 \%$ ) for three typical $\kappa$ values, namely, $\kappa=0.66,1.45$ and 3.35 . We observe that $u^{*}$ increases as $\kappa$ decreases, in agreement with reported results (Dias and Payatakes, 1986b; Hinkley et al., 1987). For relatively large solitary ganglia $\left(v^{*}>10\right)$, where the capillary forces opposed to the motion are small compared with the viscous forces, $u^{*}$ tends asymptotically to the value $1 / \kappa$ (dashed lines in Fig. 2) in accordance with the predictions of Payatakes and Dias (1984). For relatively small solitary ganglia, where the capillary forces are not negligible, $u^{*}$ is significantly smaller than its asymptotic value, again as predicted in the above reference.

The same behavior ( $\mathrm{u}^{*}$ increases as $\mathrm{\kappa}$ decreases) is also observed for the velocities of the ganglia that are members of a dense ganglion population (Fig. 2; for $S_{w}<1$ only the fitting curves are shown). For interacting ganglion populations, $\mathrm{u}^{*}$ depends on the value of $S_{w}$ significantly. This behavior can be explained by considering the effect of the water saturation, $S_{w}$, on the values of the local pressure gradient. As $S_{w}$ decreases, the portion of the porous medium through which water flows decreases, and therefore the interstitial velocity and the local pressure gradient increase (for $\mathrm{Ca}=$ constant). This, in turn, causes the increase of the ganglion velocities. Fig. 2 shows that for $S_{w}=0.7$ and in many cases for $\mathrm{S}_{\mathrm{w}}=0.6$, the reduced ganglion velocity, $\mathrm{u}^{*}$, is smaller than that of solitary ganglia. This can be explained as follows. Ganglia that are members of an interacting population have to bypass other ganglia (stranded ganglia or ganglia moving with lower velocities) during their migrations. Therefore, they move not only in the direction of the macroscopic flow, but also in the lateral directions, a fact that reduces their velocity in the main direction. This phenomenon intensifies for denser populations (say, $\mathrm{S}_{\mathrm{w}}<0.5$ ). However, in these cases, the sharp increase of $\mathrm{u}^{*}$ due to the increase of the local pressure gradient more than compensates for this phenomenon.

\section{Stranding and breakup of ganglia}

The method followed for the calculation of the stranding and breakup coefficients is similar to that proposed by Dias and Payatakes (1986b) (see also Valavanides et al., 1996). For a given set of the system parameter values $\left(\kappa, \mathrm{S}_{\mathrm{w}}\right.$, and $\mathrm{v}^{*}$; Ca, Co, $\theta_{\mathrm{a}}^{0}$ and $\theta_{\mathrm{r}}^{0}$ arc kept fixed in this study) the method includes the following steps:

- Record the lengths of ganglion migrations before the ganglia become stranded, or break, or coalesce.

- Superpose the starting planes of all migrations considered.

- Count the number of the moving ganglia at several positions, $\mathbf{x}$, in the direction of the macroscopic flow. the number of ganglia which became stranded in the interval $(x-\Delta x / 2, x+\Delta x / 2)$ (here, $\Delta x /=0.02)$ and the number of ganglia fissioned in the same interval. The following variables are then obtained:

$$
\begin{aligned}
& \chi=\frac{n r \text { of initial } v \text {-ganglia moving at position } \mathrm{x}}{\text { initial number of } v \text {-ganglia }} \ldots \ldots \ldots \ldots . . . . . . \\
& \text { initial number of } \mathrm{v} \text {-ganglia }
\end{aligned}
$$

$$
(1)=\frac{n r \text { of initial } v \text {-ganglia fissioned in the interval }\left(x \pm \frac{1}{2} \Delta x\right)}{\text { initial number of } v \text {-ganglia }}
$$

Then, the stranding coefficient, $\lambda^{\prime}$, and the breakup coefficient, $\phi^{\prime}$, (Payatakes et al., 1980) can be calculated from:

$$
\begin{aligned}
& \lambda^{\prime} l=\frac{\psi l}{\chi \Delta x} \ldots \ldots \ldots . . \text { at fixed } . / I \\
& \phi^{\prime} I=\frac{a)}{\chi \Delta x} \frac{I}{\chi \ldots . . . . . . a t ~ f i x e d ~} . v / l
\end{aligned}
$$

These coefficients express the rates of stranding and breakup of moving v-ganglia as functions of the migration length. To make these coefficients independent of $x$, their averaged values are calculated, that is, $\lambda\left(v^{*}\right)=\left\langle\lambda^{\prime}\left(v^{*}, x\right)\right\rangle$ and $\phi\left(v^{*}\right)=\left\langle\phi^{\prime}\left(v^{*}, x\right)\right\rangle$. During this calculation, the values of $\lambda^{\prime}$ and $\phi^{\prime}$ are weighted using as weights the number of ganglia at each position $x$ (or equivalently the values of $\psi$ and (1)). Then, the appropriate fitting functions $\lambda\left(\mathrm{v}^{*}\right)$ and $\phi\left(\mathrm{v}^{*}\right)$ are determined with weighted least square fitting, using the nonnalized number of ganglia of each class as weights.

The dependence of the reduced stranding coefficient, $\lambda\left(v^{*}\right) l$, and the reduced breakup coefficient, $\phi\left(v^{*}\right) l$, on water saturation, $S_{w}$, are shown in Figs. 3 and 4 for three typical $\kappa$ values, namely, $\kappa=0.66,1.45$, and 3.35 $\left(\mathrm{Ca}=10^{-4}, \mathrm{Co}_{0}=0.15, \theta_{\mathrm{a}}^{0}=45^{\circ}\right.$, and $\left.\theta_{\mathrm{r}}^{0}=35^{\circ}\right)$. In Fig. 4 the data points and the confidence intervals (confidence 

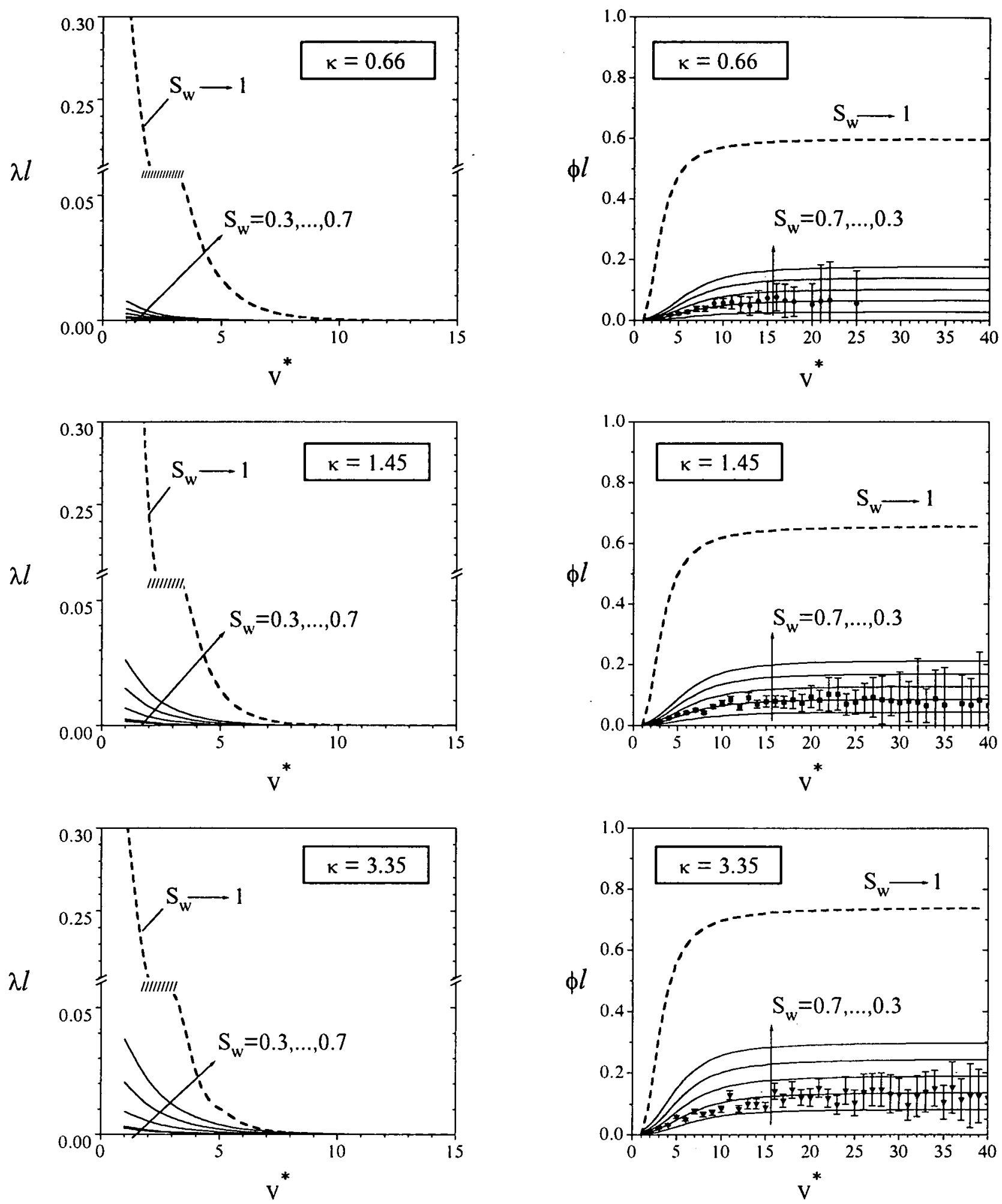

Figure 3: Dependence of the reduced stranding coefficient, $\lambda$, on the ganglion volume, $\mathrm{v}^{*}$, and water saturation, $S_{w}$, for three typical $k$ values, namely, $0.66,1.45$, and 3.35 $\left(\mathrm{Ca}=10^{-4}, \mathrm{Co}=0.15, \theta_{\mathrm{a}}^{0}=45^{\circ}\right.$, and $\left.\theta_{\mathrm{r}}^{0}=35^{\circ}\right)$.

Figure 4: Dependence of the reduced breakup coefficient, $\phi l$, on the ganglion volume, $\mathrm{v}^{*}$, and water saturation, $S_{w}$, for three typical $\kappa$ values, namely, $0.66,1.45$, and $3.35 \quad\left(\mathrm{Ca}=10^{-4}\right.$, $\mathrm{Co}=0.15, \theta_{\mathrm{a}}^{0}=45^{\circ}$, and $\theta_{\mathrm{r}}^{0}=35^{\circ}$ ). The data points and the confidence intervals (confidence level 95\%) are shown for $\mathrm{S}_{\mathrm{w}}=0.6$. 
level $95 \%$ ) are shown for $\mathrm{S}_{\mathrm{w}}=\mathbf{0 . 6}$ and all three $\mathrm{k}$ values. We observe that the values of both coefficients, $\lambda$ and $\phi$, for solitary ganglia $\left(\mathrm{S}_{\mathrm{w}} \rightarrow 1\right)$ are significantly larger than those calculated for interacting ganglion populations. Our simulations show that solitary ganglia fission before they take a "cruising" shape (Rapin, 1980; Hinkley et al., 1987). When the ganglion population is quite sparse, the small ganglia $\left(v^{*}<5\right)$ become stranded due to the relatively large value of $\lambda$, whereas larger ganglia $\left(v^{*}>5\right)$ break frequently due to the relatively large value of $\phi$ and the small daughter ganglia become stranded where they are formed. This explains why all the ganglia become stranded if the ganglion population is quite sparse, even if the capillary number is $\mathrm{Ca} \sim 10^{-4}$. When the ganglion population is dense, collisions between ganglia happen frequently. Let us consider a relatively large moving ganglion colliding with a relatively small ganglion, which is on the verge of becoming stranded. If coalcscence between these two ganglia does not take place, the large ganglion pushes the small one to move downstream, and therefore, stranding is prevented and $\lambda$ decreases. Since this phenomenon intensifies as $S_{w}$ decreases, $\lambda /$ decreases as $S_{w}$ decreases. On the other hand, as $S_{w}$ decreases, the reduced breakup coefficient, $\phi\left(\mathrm{v}^{*}\right) J$ increases for a dense ganglion population. As we explained above, as $S_{w}$ decreases, the interstitial velocity of water increases and the local pressure gradient becomes relatively large. These conditions favor dynamic breakup of the ganglia. Besides, for relatively small $S_{w}$ values, moving ganglia have to bypass other ganglia (stranded ganglia, ganglia moving with lower velocity) along their way. Hence, as $\mathrm{S}_{\mathrm{w}}$ decreases, ganglia tend to flow not only parallel to the direction of the macroscopic flow, but also in the lateral directions. This type of motion causes changes of the ganglion shape and leads to the formation of oil threads which often rupture intensifying breakup through pinchoff. Consequently, both mechanisms for ganglion breakup, that is, dynamic breakup and pinch-off intensify as $S_{w}$ decreases (keeping the rest parameters constant).

Our results (Figs. 3 and 4 ) also show that as $\kappa$ increases both $\lambda$ and $\phi$ increase, in agreement with previous works (Dias and Payatakes, 1986b). This behavior can be explained as follows (Constantinides and Payatakes, 1996). For $\kappa<1$, ganglia take a long and slender shape and follow flowpaths that are mainly parallel to the direction of the macroscopic flow. Under these conditions, the maximum length of the ganglion projected in the direction of the macroscopic flow, $L_{g}$, is relatively long. The viscous force which is responsible for ganglion mobilization is proportional to $\mathrm{L}_{\mathrm{g}}$ and the local pressure gradient (Ng and Payatakes, 1980). Hence, for $\kappa<1$, the viscous force exerted on each ganglion is relatively large and, therefore, $\lambda$ is relatively small. Moreover, the long and slender. shape of these ganglia reduces the probability of fissioning, and thus, the value of $\phi$ is relatively small. For $\kappa>1$, the ganglia show a tendency to follow flowpaths composed of relatively large pores to reduce viscous dissipation. This tendency intensifies as $\kappa$ increases. Under these conditions, $L_{g}$ decreases and ganglia flow not only in the direction of the macroscopic flow, but also in the lateral directions. As explained above this type of motion increases the stranding and breaking coefficients.

\section{Mode of ganglion breakup}

An important parameter in ganglion population dynamics is the breakup mode probability, $W(w, v) \Delta v$, expressing the probability that a moving w-ganglion will break into two daughter ganglia, one of which is a v-ganglion (Dias and Payatakes, 1986b).

The simulations for solitary ganglia have shown that the viscosity ratio, $\kappa$, does not affect $W(w, v) \Delta v$ significantly. For this reason a single fitting function $W(w, v) \Delta v$ was used for all $\kappa$ values examined (Fig. 5a). These results show that dynamic breakup is mainly responsible for the fissioning of relatively small ganglia $\left(v^{*}<10\right)$, whereas pinch-off is mainly responsible for the fissioning of relatively large ganglia. In the first case, the two daughter ganglia have approximately equal volumes, whereas in the second one, the two daughter ganglia have significantly different volumes.

The steady-state ganglion dynamics simulations have also shown that the water saturation. $S_{w}$, and the viscosity ratio, $\kappa$, do not affect $W(w, v) \Delta v$ significantly. Hence, a single fitting function $W(\mathrm{w}, v) \Delta v$ was used for all $\kappa$ and $S_{w}$ values examined (Fig. 5b). As before, the main mechanism for relatively small ganglion breakup is dynamic breakup, whereas for relatively large ganglia it is pinch-off.

Comparing $W(w, v) \Delta v$ for $S_{w} \rightarrow 1$ and $S_{w}<1$, we observe that breakup due to pinch-off intensifies for solitary ganglia. This can be explained considering that for $S_{w} \rightarrow 1$ the interstitial velocity and the local pressure gradient have relatively small values, whereas as $S_{w}$ decreases their values increase (see above) and dynamic breakup is favored. 

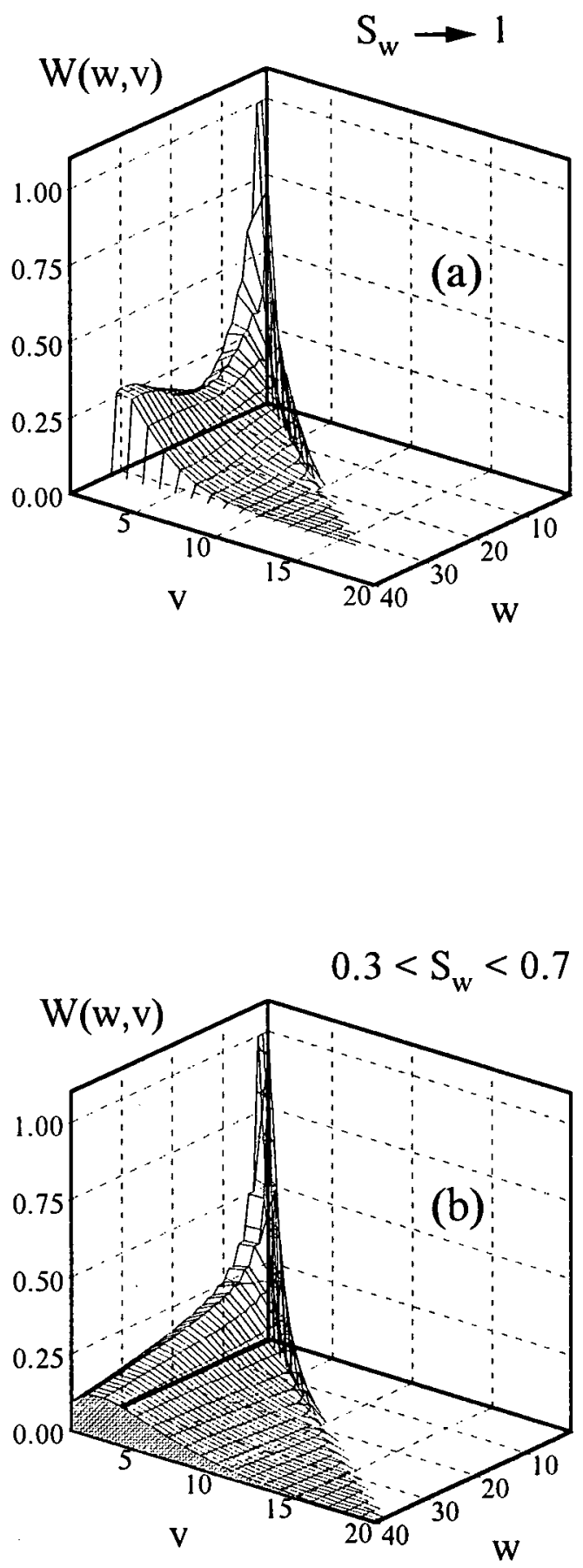

Figure 5: The mode of ganglion breakup, $W(w, v) \Delta v$ : a) for solitary ganglia, and $b$ ) for an interacting ganglion population with $0.3 \leq \mathrm{S}_{\mathrm{w}} \leq 0.7$ $\left(\mathrm{Ca}=10^{-4}, \mathrm{Co}=0.15, \theta_{\mathrm{a}}^{0}=45^{\circ}\right.$, and $\left.\theta_{\mathrm{r}}^{0}=35^{\circ}\right)$.

\section{CONCLUSIONS}

A recently developed computer-aided threedimensional network simulator was used to predict the flow behavior of solitary ganglia and that of ganglia which are members of an interacting ganglion population. The simulator is used to predict the differences of ganglion velocities, stranding and breakup coefficients and mode of ganglion breakup as functions of the ganglion volume, the water (or oil) saturation and the viscosity ratio. The main conclusions of this study are the following:

- The reduced mean time-averaged ganglion velocity, $\mathbf{u}^{*}$, is a strong function of the water saturation, $S_{w}$, and the viscosity ratio, $\kappa$. It increases as $S_{w}$ and/or $\kappa$ decreases.

- The values of the stranding, $\lambda$, and the breakup, $\phi$, coefficients are relatively large for solitary ganglia $\left(S_{w} \rightarrow 1\right)$, but decrease drastically in the case of dense ganglion populations. For dense populations, as the water saturation, $S_{w}$, decreases, the stranding coefficient, $\lambda$, decreases. The behavior of $\phi$ is more complex. As $S_{w}$ decreases from 1 to $\sim 0.7, \phi$ drops sharply, but as $S_{w}$ continues to decrease in the range 0.7 to $0.3, \phi$ increases again markedly, (without reaching however the high value corresponding to $\left.S_{w} \rightarrow 1\right)$. Both coefficients increase as the viscosity ratio, $\kappa$, increases.

- The mode of ganglion breakup is almost independent of the value of the water saturation, $S_{w}$, and the viscosity ratio, $\kappa$.

\section{NOMENCLATURE}

Latin symbols

Ca capillary number

Co coalescence factor

Gm ganglion mobilization number

$\mathrm{k}_{\mathrm{rw}} \quad$ relative permeability to water

I mean node-to-node distance of the pore network

$\mathrm{L}_{\mathrm{g}}(\mathrm{v}) \quad$ mean maximum length of $\mathrm{v}$-ganglia projected in the direction of the macroscopic flow

$r \quad$ oil/water flowrate ratio

$\mathrm{S}_{\mathrm{o}} \quad$ oil saturation

$\mathrm{S}_{\mathrm{w}} \quad$ water saturation

$t$ time

$\bar{u} \quad$ time-averaged velocity with which the centroid of a ganglion moves

$<\overline{\mathrm{u}}>$ (v) mean time-averaged velocity of v-ganglia

$\mathrm{U}_{\mathrm{w}} \quad$ superficial velocity of water

$\mathrm{w}, \mathrm{v} \quad$ ganglion volumes 
$\mathrm{V}_{\mathrm{CEVS}}$ mean volume of a chamber and its adjacent half throats

$\mathrm{W}(\mathrm{w}, \mathrm{v}) \Delta$ vprobability that a moving w-ganglion will break into two daughter ganglia, one of which is a v-ganglion

$\mathrm{x}, \mathrm{y}, \mathrm{z} \quad$ Cartesian coordinates

\section{Greek symbols}

$\varepsilon \quad$ porosity

$\theta_{\mathrm{a}}, \theta_{\mathrm{r}}$ dynamic advancing and receding contact angles

$\kappa \quad$ oil/water viscosity ratio

$\lambda^{\prime}, \lambda \quad$ stranding coefficients

$\varphi^{\prime}, \varphi \quad$ breakup coefficients

$\chi, \psi, \omega$ variables defined in Eqs. (1), (2) and (3), respectively

\section{Superscripts}

* dimensionless variable

\section{Acknowledgments}

This work was supported by the Institute of Chemical Engineering and High Temperature Chemical Processes, ICE-HT/FORTH.

\section{REFERENCES}

1. Avraam, D.G., Kolonis, G.B., Roumeliotis, T.C., Constantinides, G.N., and Payatakes, A.C. 1994 Steady-state two-phase flow through planar and nonplanar model porous media. TiPM 16, 75.

2. Avraam, D.G., and Payatakes, A.C. 1995 Flow regimes and relative permeabilities during steadystate two-phase flow in porous media, J. Fluid Mech., 293, 207

3. Constantinides, G.N., and Payatakes, A.C. 1989 A three dimensional network model for consolidated porous media. Basic studies, Chem. Eng. Comm. 81, 55

4. Constantinides, G.N., and Payatakes, A.C. 1991 A theoretical model of collision and coalescence of ganglia in porous media. J. Coll. Int. Sci. 141, 486.

5. Constantinides, G.N., and Payatakes, A.C. 1996 Network simulation of steady-state two-phase flow in consolidated porous media AIChE J. 42, 369.

6. de la Cruz, V., and Spanos, T.J.T. 1983 Mobilization of oil ganglia AIChE J. 29, 854

7. Dias, M.M., and Payatakes, A.C. 1986a Network models for two-phase flow in porous media. Part 1.
Immiscible microdisplacement of non-wetting fluids. J. Fluid Mech. 164, 305.

8. Dias, M.M., and Payatakes, A.C. 1986b Network models for two-phase flow in porous media. Part 2. Motion of oil ganglia. J. Fluid Mech. 164, 337.

9. Dullien, F.A.L., and Dhawan, G.K. 1975 Bivariate pore-size distribution of some sandstones, $J$. Colloid Interface Sci. 52, 129.

10. Hinkley, R.E., Dias M.M., and Payatakes, A.C. 1987 On the motion of oil ganglia in porous media. Physicochemical Hydrodynamics 8, 185

11. Legait, B. 1983 Laminar flow of two phases through a capillary tube with variable square cross-section. $J$. Colloid Interface Sci. 96, 28

12. Legait, B., Sourieau, P., and Combarnous, M. 1983 Inertia, viscosity, and capillary forces during twoPhase flow in a constricted capillary tube $J$. Colloid Interface Sci. 91, 400

13. Melrose, J.C., and Brandner, C.F. 1974 Role of capillary forces in determining microscopic displacement efficiency for oil recovery by water flooding J. Can. Petr. Tech. 13, 54

14. Ng, K.M., Davis, H.T., and Scriven, L.E. 1978 Visualization of blob mechanisms in flow through porous media Chem. Eng. Sci. 33, 1009

15. Ng, K.M., and Payatakes, A.C. 1980 Stochastic simulation of the motion, breakup and stranding of oil ganglia in water-wet granular porous media during immiscible displacement. $A J C h E J .26,419$.

16. Payatakes, A.C. 1982 Dynamics of oil ganglia during immiscible displacement in water-wet porous media. Ann. Rev. Fluid Mech. 14, 365.

17. Payatakes, A.C., and Dias, M.M. 1984 Immiscible microdisplacement and ganglion dynamics in porous media. Rev. Chem. Eng. 2, 85.

18. Payatakes, A.C., Ng, K.K., and Flumerfelt, R.W. 1980 Oil ganglion dynamics during immiscible displacement: Model formulation. AJChE J. 26, 430 .

19. Rapin, S. 1980 Behaviour of non-wetting oil ganglia displaced by an aqueous phase M.S. Thesis, Univ. of Houston, Houston, Texas.

20. Sahimi, M. 1993 Flow phenomena in rocks: From continuum models to fractals, percolation, cellular automata, and simulating annealing. Rev. Mod. Phys. 65, 1393

21. Valavanides, M.S., Constantinides, G.N., and Payatakes, A.C. 1996 Macroscopic theory of twophase flow in porous media based ganglion dynamics. submitted in J. Flujd Mech.

22. Vizika, O., Avraam D.G., and Payatakes, A.C. 1994 On the role of the viscosity ratio during low capillary number forced imbibition in porous media. J. Colloid Interface Sci. 165, 386 